\title{
Application of Fuzzy DEMATEL in Risks Evaluation of Knowledge-Based Networks
}

\author{
M. Abbasi, R. Hosnavi, and B. Tabrizi \\ Department of Management and Soft Technologies, Malek-e Ashtar University of Technology, Tehran, Iran \\ Correspondence should be addressed to B. Tabrizi; btabrizi_maut@yahoo.com
}

Received 28 June 2013; Revised 30 October 2013; Accepted 2 November 2013

Academic Editor: F. Hosseinzadeh Lotfi

Copyright ( $) 2013$ M. Abbasi et al. This is an open access article distributed under the Creative Commons Attribution License, which permits unrestricted use, distribution, and reproduction in any medium, provided the original work is properly cited.

\begin{abstract}
Developing new products has received much attention within the last decades. This issue can be highlighted for strategic innovations, in particular. Recently, knowledge-based networks have been introduced in order to facilitate the affair of transforming knowledge into commercial products which can be regarded as a set of research centers, universities, knowledge intermediaries, customers, and so forth. However, there is a wide range of risk factors that are liable to affect the chain performance. Hence, this paper aims to consider the most influencing criteria that can play a more significant role in achievements of such networks. To do so, DEMATEL has been applied to take the relationships between the risk factors into account. Moreover, fuzzy set theory has been utilized in order to deal with the linguistic variables. Finally, the most important factors are identified and their relations are determined.
\end{abstract}

\section{Introduction}

Over the last years, companies have extensively concentrated on developing high quality products and services which can be justified with respect to the rise of consumer demands and severe competition amongst the companies. The competition is more highlighted about the case of introducing novel products, in particular [1].

It is commonly observed that many companies are hesitant to endure the risks of experiencing innovations or adopting leading-edge technologies, particularly "strategic" innovations. The strategic innovations are expensive with a noticeable proportion of ambiguity, which make the associated research and development take longer. The risks can be facilitated by the application of collaboration strategies. However, the cooperation may be hindered when the companies look for collaborating with technology partners that are reluctant to have serious working relations. Technological innovations may be followed by another shortcoming: the intrinsic risks with science-based innovations which are often hard to express, define, and quantify.

The organizations may encounter significant financial and opportunity losses if the development or launch of their new product is deferred. For instance, Kurawarwala and
Matsuo [2] showed that a six-eight-month delay in launch of products such as computers and cellular phones by a computer manufacturer results in a 50-75\% loss in revenue. In a more recent study, McGrath and MacMillan [3] indicated that a six-month delay in the introduction of the product leads to a $\$ 2$ million decrease in the project's net present value. These instances can prove the importance of enhancing new product development capabilities, increasing efficiency, and reducing development costs.

According to the extant literature, it can be stated that research on knowledge-based networks $(\mathrm{KN})$ chiefly concentrates on concepts, mechanism, and components. KNs can be grouped in terms of three levels $[4,5]$ as follows.

(1) The macrolevel which addresses activities raising innovation at a national level [6].

(2) The medium level which deals with the companies' business activities that are centered within knowledge flows [7].

(3) The microlevel that incorporates the activities between functional areas [8].

The idea behind KNs can be tracked similar to the ordinary supply chain management. In other words, a typical 
knowledge chain aims at matching supplies to customer demand like the product supply chains. As a general rule, the KNs can be exemplified by universities, knowledge intermediaries, research institutions, producers, retailers, customers, and so forth. The KNs encompass the entire process of acquiring, making, or transferring knowledge [1]. Although "knowledge" is characterized in the form of data or information, the flow unit can be regarded as the potential value that can be added to a company, which in turn triggers or creates focused action. Consequently, it is intended here to enhance the overall performance of the KNs, transforming knowledge into commercially available products, by extension of the common view of companies exclusively focusing on physical, information or financial supply chains. "Knowledge" points to the particular information in universities and companies that can lead to product or process innovation.

Recent research in supply and operations management also highlights the value of knowledge as a strategic resource. For example, Hult et al. [9] analyze the intangible aspects of why some supply chains outperform while others brawl. In a research study, Craighead et al. [10] showed that the performance is influenced by the degree of knowledge development capacity and intellectual capital efforts amongst a remarkable number of companies, that is, 489 companies.

From a broader point of view, a given $\mathrm{KN}$ can be affected by different kinds of risks. These risks include a varied set of issues which can potentially impede the appropriate achievements of the chain members. Hence, it is necessary to devote a substantial attention towards the consideration of the risks and selection of the most important ones. However, there is unfortunately a bare literature on the aforementioned issue, to the best knowledge of the authors. Therefore, we have tried to identify the most significant risk factors so that the mitigation phase can be followed more purposefully. In other words, the paper aims to investigate the most influencing risk factors threatening the performance of the KNs. The issue has been taken into account by the DEMATEL technique in which fuzzy set theory has dealt with the imprecise judgments.

The rest of this paper is organized as follows. Problem definition is presented in Section 2, in which the risk factors are categorized. Furthermore, fuzzy DEMATEL is described in order to deal with the factors evaluation. An empirical study is carried out to show the applicability of the given method in Section 3. Finally, conclusions and future research directions are addressed in Section 4.

\section{Problem Definition}

In this section, the factors that can affect the KNs performance are explained first. Afterwards, risk sources and different types of risks are taken into consideration. To describe how the innovation process is influenced by several factors, first, it can be stated that first dynamic factors push the innovation process. The forces could be stemmed from market demand, technological progress, government initiatives, and so forth. Moreover, the success of the innovation process depends on a range of supportive functions such as the environmental system, market system, and technology availability. Finally, one has to take political and governmental factors into account which can yield in assistance and guidance on each level of the economy levels.

Over all, major processes include (1) identifying knowledge demand and selection of knowledge sources, (2) sharing and transferring knowledge, and (3) applying knowledge to convert it into innovative products and processes. It is evident that risk analysis reveals both science and politics.

Supply risk can be highlighted as one of the chief categories influencing supply and operations management which has an enriched body of literature (e.g., [11, 12]). On the other hand, a significant literature exists on definitions of risk and uncertainty on, for instance, assessing technological risks [13], mitigating technological risks, and communicating technological risks through knowledge-based stakeholder information systems [14].

Amongst different papers that have addressed risk consideration frameworks for KNs, Lindroth and Norman [15] proposed a risk framework in accordance with experiences in the telecommunications sector with three facets including unit, risk type, and risk control. The proposed risk management framework of Christopher [16] comprises of four stages with respect to risk component definitions. Kulkarni et al. [17] considered the dynamics of supply networks and the need to investigate network complexity and feedback mechanisms.

Here, we have applied different risk types proposed by Xiwei et al. [1] in which they first collected real-world examples and cases from the existing literature and then analyzed expert interviews and discussions. According to their risk categorization, the given framework has been divided into eight risk types. The main types of risks are mentioned in Table 1.

A brief definition of fuzzy set theory is addressed here in order to deal with imprecise judgments, in addition to DEMATEL method. Afterwards, fuzzy DEMATEL is described to be applied in the case study.

2.1. Fuzzy Set Theory (FST). FST was introduced by Zadeh [18] based on the concept of membership function to take linguistic variables into account. The theory tries to consider uncertain data which can be associated with existing fuzziness of peoples' thoughts and perceptions. It should be noted that the results are in such circumstances that are extremely affected by subjective judgments. The FST has proved efficient in coping with vagueness of human thought and expression while decision-making. A linguistic variable can be defined as a variable whose values are assigned with respect to phrases or sentences in a natural language. The linguistic terms help the DMs state their judgments and assessments easily as it can be represented by different fuzzy-shaped numbers (e.g., triangular or trapezoidal).

It is presumed here that linguistic values can be efficiently represented by triangular fuzzy numbers (TFNs). A TFN $\widetilde{N}$ can be defined as a triplet $(l, m, u)$, shown by Figure 1 . The 
TABLE 1: Main types of risks threatening KNs.

\begin{tabular}{ll}
\hline Risk type & Instances \\
\hline Policy risk & $\begin{array}{l}\text { Governmental policies, changes in the law, bureaucracy, lack of decision support, } \\
\text { new regulations, economic shifts, lack of formal procedures, lack of quality control } \\
\text { system, recession, inflation, increasing labor costs, actions and sanctions, shifts in } \\
\text { legislation, change in exchange rates, lack of capital, labor disputes, inaccurate } \\
\text { forecasts, infrastructure breakdown, and so forth. }\end{array}$ \\
\hline Market risk & $\begin{array}{l}\text { Changes in customer expectations, price changes, intensified competition, } \\
\text { variations in customer demand, supplier bankruptcy, uncertainty about } \\
\text { competitors' moves, supply disruption, and so forth. }\end{array}$ \\
\hline Culture risk & $\begin{array}{l}\text { Cultural differences between companies and people, lack of trust, language barriers, } \\
\text { less willingness to share knowledge, misunderstandings, and so forth. }\end{array}$ \\
\hline Knowledge risk & $\begin{array}{l}\text { Cultural differences between companies and people, lack of trust, language barriers, } \\
\text { less willingness to share knowledge, misunderstandings, and so forth. }\end{array}$ \\
\hline Cooperative risk & $\begin{array}{l}\text { Organizational barriers, inefficient processes, lack of coordination, bureaucracy, } \\
\text { natural disasters, and so forth. }\end{array}$ \\
\hline Contractual risk & $\begin{array}{l}\text { Renegotiation of contracts, leave of business partners, supplier bankruptcy, poor } \\
\text { quality materials, opportunism, lack of formal procedures, and so forth. }\end{array}$ \\
\hline Knowledge asset risk & $\begin{array}{l}\text { Lack of technology, changes in technology, patent right infringement, theft, forging, } \\
\text { and so forth. }\end{array}$ \\
\hline Talent risks & \begin{tabular}{l} 
Fluctuation of skillful employees, lack of human resources, and so forth. \\
\hline
\end{tabular}
\end{tabular}

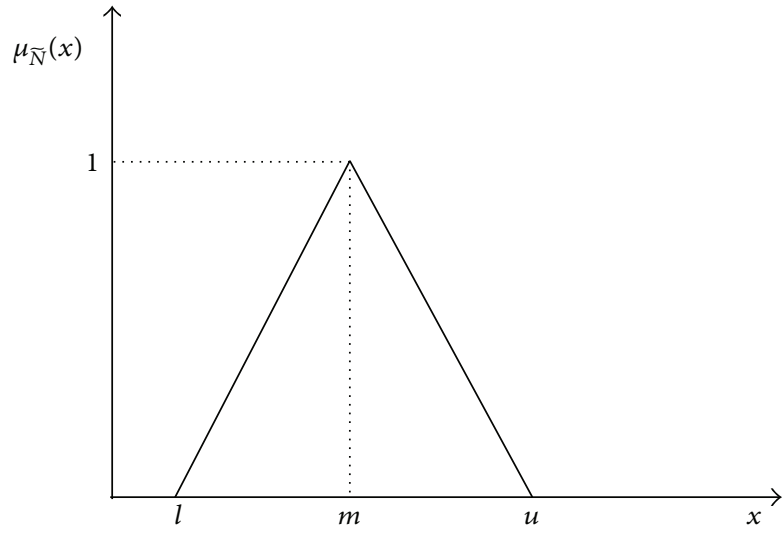

FIGURE 1: A symmetric TFN.

associated membership function $\mu_{\widetilde{N}}(x)$ is calculated by (1), in which $l, m$, and $u$ are real numbers and $l \leq m \leq u$. Consider the following:

$$
\mu_{\widetilde{N}}(x)= \begin{cases}0, & x<l, \\ \frac{(x-l)}{(m-l)}, & l \leq x \leq m, \\ \frac{(u-x)}{(u-m)}, & m \leq x \leq u, \\ 0, & x>u .\end{cases}
$$

2.2. DEMATEL. Decision Making Trial and Evaluation Laboratory (DEMATEL) was first introduced by the Battelle Memorial Institute through its Geneva Research Centre [19, 20]. DEMATEL can be interpreted as an extended method in setting and analyzing a structural model from which the relation between complex criteria can be extracted. The original DEMATEL method aimed to find integrated solutions to incompatible societies around the world. Afterwards, it could receive much attention as it proved to be practical in determining intricate causal relationships.

DEMATEL method is based on digraphs and can separate promising factors in regard to two cause and effect groups. Digraphs can be obtained from DEMATEL, interpreted as directed graphs, letting the user be informed about the directed relationships of the subsystem. In other words, a digraph may typically characterize a communication network or some domination relation between the factors. Furthermore, DEMATEL discloses the importance of the given factors and determines how a particular factor influences over other ones, on the other hand.

A notable advantage of the method is to moderate the number of criteria under consideration. The end product of this methodology is a visual sketch by which the actions and decisions can be made. Many organizations utilize DEMATEL to determine key factors so that they can enhance their organization performance [21].

The implementation steps of DEMATEL are concisely described as follows.

(1) Find the pair-wise comparison matrix: in the first step, the decision-maker (DM) determines the extent to which a specific criterion affects another one. In other words, a pair-wise comparison matrix is developed by the DM in which array $a_{i j}$ represents how criterion $i$ influences over criterion $j$. All comparisons are performed regarding an integer score ranging from $0,1,2,3$, and 4 . The scores stand for "no influence," "very low influence," "low influence," "high influence," and "very high influence," respectively. An $n \times n$ matrix is developed like $\mathbf{A}=\left[a_{i j}\right]_{n \times n}$, 
while the comparisons are all performed. However, the given matrix should be extended to an average one in the presence of a group of experts. Equation (2) shows the formula of a typical average matrix, in which $H$ denotes the number of experts. Consider the following:

$$
\left[a_{i j}\right]_{n \times n}=\frac{1}{H} \sum_{k=1}^{H}\left[X_{i j}^{k}\right]_{n \times n} .
$$

Without loss of generality, the product is also taken as the initial direct relation matrix. It displays the initial direct influences that an element dispatches to and receives from other elements. Moreover, the causal effect between each pair of criteria can be mapped out by developing an influence map. A typical network influence map is illustrated by Figure 2. The letters and arrows denote criteria of the considered system and the effects, respectively. For instance, the arrow from $b$ to $c$ shows the effect that $b$ practices on $c$ and number 3 states that the strength of the effect is high.

(2) Calculate the normalized initial direct-relation matrix: the normalized direct-relation matrix $\mathbf{M}$ can be obtained by (3) with regard to the direct-relation matrix A. It should be noted that all diagonal elements are set equal to zero. Consider the following:

$$
\begin{gathered}
\mathbf{M}=k \cdot \mathbf{A}, \\
k=\frac{1}{\max \sum_{j=1}^{n}\left|a_{i j}\right|} ; \quad \forall i=1,2, \ldots, n .
\end{gathered}
$$

(3) Compute the total relation matrix: the total-relation matrix $\mathbf{S}$ can be calculated while the normalized direct-relation matrix is obtained. $\mathbf{S}$ can be derived by (4), in which I stands for the identity matrix. Consider the following:

$\mathbf{S}=\mathbf{M}+\mathbf{M}^{2}+\mathbf{M}^{3}+\cdots+\mathbf{M}^{\infty}=\sum_{i=1}^{\infty} \mathbf{M}^{i}=\mathbf{M}(1-\mathbf{M})^{-1}$.

(4) Compute dispatcher and receiver group: dispatcher and receiver groups can be identified regarding the values of $\mathbf{D}-\mathbf{R}$ and $\mathbf{D}+\mathbf{R}$, in which $\mathbf{R}$ is the sum of columns and $\mathbf{D}$ is the sum of rows of matrix $\mathbf{S}$. Equation (5) defines a level of influence to others and a level of relationships with others. D - $\mathbf{R}$ indicates how a given criterion influences over the other ones. The criteria with positive value, called dispatcher, practice more influence and are therefore presumed to have higher priorities. On the contrary, the criteria with negative values, called receiver, are mostly influenced by the others and are expected to have lower priorities. On the other hand, the degree of relation between a given criteria with others can be identified from $\mathbf{D}+\mathbf{R}$. The criteria with higher

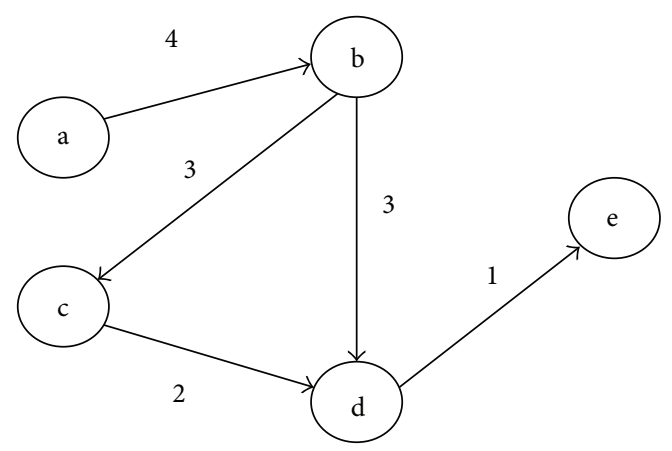

FIgURE 2: An influence map.

values represent more amounts of relationships with others while the lower values imply fewer amounts of relationships with others. Consider the following:

$$
\begin{aligned}
\mathbf{S}=\left[s_{i, j}\right]_{n \times n} ; \quad i, j \in\{1,2, \ldots, n\}, \\
\mathbf{D}=\sum_{j=1}^{n} s_{i, j}, \\
\mathbf{R}=\sum_{i=1}^{n} s_{i, j} .
\end{aligned}
$$

(5) Set a threshold value and obtain the impact-diagraphmap: the DM is required to select a threshold value for the influence level to obtain an appropriate impactdiagraph-map. The elements that exceed the set threshold are picked out and converted into the map. The threshold value is selected with respect to the DM or experts' beliefs and the impact-diagraph-map can be provided by mapping the data set of $(\mathbf{D}+\mathbf{R}, \mathbf{D}-\mathbf{R})$. $\mathbf{D}+\mathbf{R}$ and $\mathbf{D}-\mathbf{R}$ are reflected by the horizontal and vertical axis, respectively.

DEMATEL is applied in this paper as an efficient method in order to build a structural model and visualize the causal relationship of subsystems through a causal diagram. The criteria with higher priorities can be isolated to be utilized in further studies aiming to evaluate different systems. However, as the linguistic judgments are fraught with different uncertainties and vagueness, it is required to consider the ambiguities. Accordingly, it is needed to integrate the DEMATEL method with fuzzy logic.

2.3. Fuzzy DEMATEL. The implementation of fuzzy DEMATEL has been discussed in this section. In fact, it is needed to determine how to practice the imprecision of given judgments in the decision-making process. To do so, an efficient fuzzy aggregation method should be utilized. Likewise, it is required to use a tailored defuzzification method so that a fuzzy number can be well characterized by its shape, spread, height, and relative location on the $x$-axis [22]. Here, we have used the converting fuzzy data into crisp scores (CFCS) defuzzification method as the fuzzy aggregation procedure. 
The adoption can be justified as the CFCS method can provide a better crisp value than other similar methods (e.g., the centroid method). The procedure of CFCS method proposed by Opricovic and Tzeng [22] is such that the left and right scores are taken into account by fuzzy min and fuzzy max, and a weighted average value is obtained as the total score with respect to the membership functions. Let us suppose that $\widetilde{z}_{i j}^{k}=\left(l_{i j}^{k}, m_{i j}^{k}, r_{i j}^{k}\right)$ stands for fuzzy assessments of evaluator $k(k=1,2, \ldots, p)$ about the degree to which the criterion $i$ influences over criterion $j$. The adopted method consists of a five-step algorithm, described as follows.

(1) Normalization:

$$
\begin{aligned}
x l_{i j}^{k} & =\frac{\left(l_{i j}^{k}-\min l_{i j}^{k}\right)}{\Delta_{\min }^{\max }}, \\
x m_{i j}^{k} & =\frac{\left(m_{i j}^{k}-\min l_{i j}^{k}\right)}{\Delta_{\min }^{\max }}, \\
x r_{i j}^{k} & =\frac{\left(r_{i j}^{k}-\min l_{i j}^{k}\right)}{\Delta_{\min }^{\max }},
\end{aligned}
$$

in which $\Delta_{\min }^{\max }=\max r_{i j}^{k}-\min l_{i j}^{k}$.

(2) Calculate left $(l s)$ and right $(r s)$ normalized values:

$$
\begin{aligned}
x l s_{i j}^{k} & =\frac{x m_{i j}^{k}}{\left(1+x m_{i j}^{k}-x l_{i j}^{k}\right)}, \\
x r s_{i j}^{k} & =\frac{x r_{i j}^{k}}{\left(1+x r_{i j}^{k}-x m_{i j}^{k}\right)} .
\end{aligned}
$$

(3) Calculate total normalized crisp value:

$$
x_{i j}^{k}=\frac{\left[x l s_{i j}^{k}\left(1-x l s_{i j}^{k}\right)+x r s_{i j}^{k} x r s_{i j}^{k}\right]}{\left[1-x l s_{i j}^{k}+x r s_{i j}^{k}\right]} .
$$

(4) Calculate total normalized crisp value:

$$
z_{i j}^{k}=\min l_{i j}^{k}+x_{i j}^{k} \Delta_{\min }^{\max }
$$

(5) Integrate the crisp values:

$$
z_{i j}=\frac{1}{p}\left(z_{i j}^{1}+z_{i j}^{2}+\cdots+z_{i j}^{p}\right) .
$$

Finally, Table 2 can be applied in order to transform the aforementioned linguistic terms into positive TFNs $\left(l_{i j}, m_{i j}, r_{i j}\right)$.

\section{Case Study and Findings}

As stated earlier, it is important to investigate the amount of influence a given criterion practices over another one and vice versa. Hence, DEMATEL method could be suggested
TABLE 2: Fuzzy linguistic scale.

\begin{tabular}{lc}
\hline Linguistic terms & TFNs \\
\hline No influence & $(0,0,0.25)$ \\
Very low influence & $(0,0.25,0.5)$ \\
Low influence & $(0.25,0.5,0.75)$ \\
High influence & $(0.5,0.75,1.0)$ \\
Very high influence & $(0.75,1.0,1.0)$ \\
\hline
\end{tabular}

TABLE 3: The assessment data of the risk types.

\begin{tabular}{ccccccccc}
\hline & C1 & C2 & C3 & C4 & C5 & C6 & C7 & C8 \\
\hline C1 & No & VH & VL & H & H & VH & L & L \\
C2 & H & No & VL & VL & VL & H & VL & No \\
C3 & No & L & No & VH & H & H & No & H \\
$C 4$ & L & No & VL & No & VH & H & VL & L \\
$C 5$ & No & H & L & VH & No & H & No & VL \\
$C 6$ & VL & L & VL & L & L & No & VL & VL \\
$C 7$ & H & VL & VL & VH & VL & L & No & H \\
$C 8$ & H & L & H & L & No & L & L & No \\
\hline
\end{tabular}

as an appropriate approach capable of highlighting the most influencing risk factors. This section aims at taking a typical instance into account to extract the most crucial factors from the ones proposed by Xiwei et al. [1].

In the first step, $C_{1}, C_{2}, \ldots, C_{8}$ are applied representing the aforementioned risk types, respectively. The opinions of a set of experts were gathered through the prepared questionnaires in order to receive the judgments. Typically, the assessment data of a given expert is shown in Table 3. Afterwards, the initial direct-relation matrix is determined regarding the whole experts' opinions by (6)-(16), shown in Table 4. The normalized direction-relation matrix is then produced through (3), illustrated in Table 5. Finally, totalrelation matrix is obtained using (4), shown in Table 6. Moreover, $(\mathbf{D}-\mathbf{R})$ and $(\mathbf{D}+\mathbf{R})$ values are computed in order to achieve the impact-diagraph-map.

On the other hand, from the causal diagram depicted in Figure 3, it can be induced that policy risk (i.e., C1) is the most crucial influencing criterion as it possesses the highest intensity of relationship to others (the issue can be investigated through the obtained values of $\mathbf{D}-\mathbf{R}$ measure). Consequently, it can be taken into consideration as the key risk criterion and factor in risk mitigation of the KNs. Likewise, the knowledge asset risk and culture risk are selected as the key risk factors with respect to the obtained strategy map. Moreover, it is needed to decide on the appropriate threshold so that the factors can be picked out that have the minimum amount of influence over the others. Those relations whose values are above the predefined threshold determine how a given factor affects or is affected by the other ones. Here, the threshold has been set equal to 0.05 . Market risk, knowledge risk, cooperative risk, contractual risk, and talent risk are also identified as the effects regarding the causal diagram. 
TABLE 4: The initial direct-relation matrix.

\begin{tabular}{ccccccccc}
\hline & $C 1$ & $C 2$ & $C 3$ & $C 4$ & $C 5$ & $C 6$ & $C 7$ \\
\hline$C 1$ & 0.000 & 0.775 & 0.517 & 0.767 & 0.794 & 0.812 & 0.574 & 0.630 \\
$C 2$ & 0.652 & 0.000 & 0.372 & 0.514 & 0.488 & 0.693 & 0.520 \\
$C 3$ & 0.191 & 0.578 & 0.000 & 0.749 & 0.740 & 0.675 & 0.241 \\
$C 4$ & 0.457 & 0.266 & 0.414 & 0.000 & 0.652 & 0.686 & 0.319 & 0.588 \\
$C 5$ & 0.276 & 0.785 & 0.550 & 0.864 & 0.000 & 0.643 & 0.177 \\
$C 6$ & 0.418 & 0.488 & 0.319 & 0.376 & 0.406 & 0.000 & 0.472 \\
$C 7$ & 0.706 & 0.288 & 0.518 & 0.682 & 0.507 & 0.546 & 0.000 & 0.557 \\
$C 8$ & 0.624 & 0.434 & 0.699 & 0.422 & 0.206 & 0.440 & 0.633 \\
\hline
\end{tabular}

TABLE 5: The normalized direct-relation matrix.

\begin{tabular}{ccccccccc}
\hline & $C 1$ & $C 2$ & $C 3$ & $C 4$ & $C 5$ & $C 6$ & $C 7$ & 08 \\
\hline$C 1$ & 0.00000 & 0.15917 & 0.106182 & 0.157527 & 0.163072 & 0.166769 & 0.117889 & 0.12939 \\
$C 2$ & 0.133908 & 0.00000 & 0.076402 & 0.105566 & 0.100226 & 0.142329 & 0.106798 & 0.042103 \\
$C 3$ & 0.039228 & 0.11871 & 0.00000 & 0.15383 & 0.151982 & 0.138632 & 0.049497 & 0.120764 \\
$C 4$ & 0.093859 & 0.054631 & 0.085028 & 0.00000 & 0.133908 & 0.140891 & 0.065517 & 0.098583 \\
$C 5$ & 0.056685 & 0.161224 & 0.11296 & 0.177449 & 0.00000 & 0.13206 & 0.036352 & 0.058328 \\
$C 6$ & 0.085849 & 0.100226 & 0.065517 & 0.077223 & 0.083385 & 0.00000 & 0.09694 & 0.114397 \\
$C 7$ & 0.144999 & 0.05915 & 0.106387 & 0.14007 & 0.104128 & 0.112138 & 0.00000 & 0.130006 \\
$C 8$ & 0.128158 & 0.089135 & 0.143561 & 0.086671 & 0.042308 & 0.090368 & 0.081125 & 0.00000 \\
\hline
\end{tabular}

TABLE 6: The total-relation matrix.

\begin{tabular}{lccccccccccc}
\hline & $C 1$ & $C 2$ & $C 3$ & $C 4$ & $C 5$ & $C 6$ & $C 7$ & $C 8$ & $\mathbf{D}$ & $\mathbf{D}+\mathbf{R}$ & $\mathbf{D}-\mathbf{R}$ \\
\hline$C 1$ & 0.00000 & 0.07846 & 0.045032 & 0.08694 & 0.08311 & 0.09622 & 0.04435 & 0.05723 & 0.4913 & 0.7227 & 0.2600 \\
$C 2$ & 0.04817 & 0.00000 & 0.023977 & 0.04281 & 0.03692 & 0.06392 & 0.03203 & 0.01219 & 0.2600 & 0.5476 & -0.0275 \\
$C 3$ & 0.01130 & 0.04503 & 0.00000 & 0.06986 & 0.06324 & 0.06319 & 0.01260 & 0.04335 & 0.3085 & 0.5516 & 0.06551 \\
$C 4$ & 0.02897 & 0.01653 & 0.026219 & 0.00000 & 0.05025 & 0.05995 & 0.01633 & 0.03155 & 0.2298 & 0.6356 & -0.176 \\
$C 5$ & 0.01663 & 0.064984 & 0.038659 & 0.08181 & 0.00000 & 0.05821 & 0.00865 & 0.01735 & 0.2863 & 0.6009 & -0.0283 \\
$C 6$ & 0.02526 & 0.03234 & 0.01829 & 0.02655 & 0.0264 & 0.00000 & 0.02591 & 0.0365 & 0.1913 & 0.6193 & -0.2366 \\
$C 7$ & 0.05728 & 0.02071 & 0.03938 & 0.06555 & 0.0417 & 0.05142 & 0.00000 & 0.0509 & 0.3270 & 0.4885 & 0.1654 \\
$C 8$ & 0.04369 & 0.02952 & 0.05150 & 0.03228 & 0.01292 & 0.03507 & 0.02163 & 0.00000 & 0.2266 & 0.4757 & -0.0224 \\
$\mathbf{R}$ & 0.23133 & 0.28760 & 0.24308 & 0.40582 & 0.31463 & 0.42799 & 0.16153 & 0.24913 & & \\
\hline
\end{tabular}

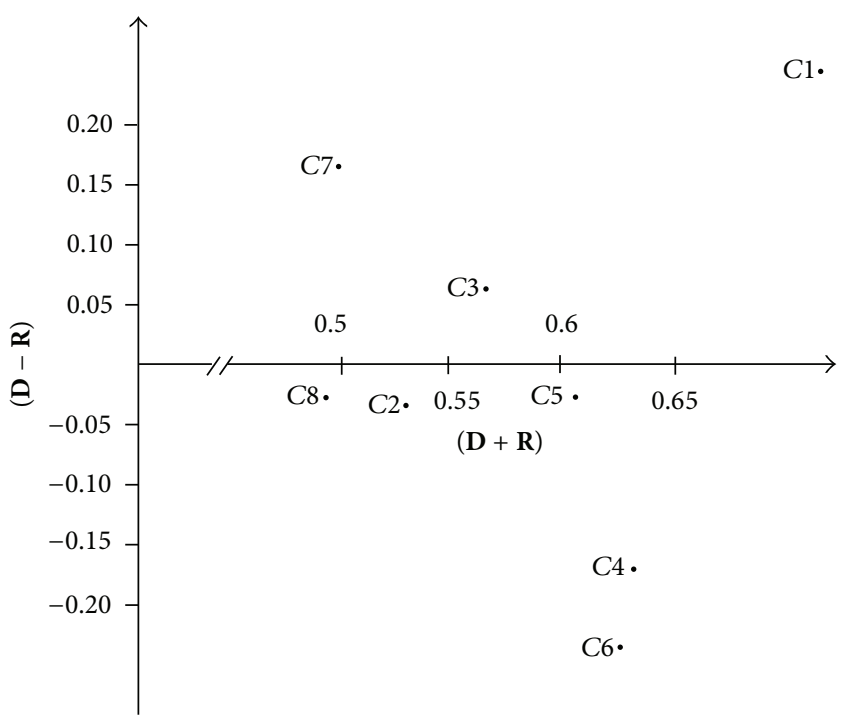

Figure 3: The causal diagram.

\section{Conclusions}

KNs have been concentrated within the last years as efficient sources of transforming the knowledge into the commercial products. They can play a significant role in satisfaction of the customers' expectations, in addition to the knowledge flow preservation along with the chain. However, the KNs may be threatened by different sets of risk factors. Hence, it is required to address the most influencing risk types so that the preventive and modification actions can be performed appropriately. This paper took the risk evaluation of such networks into account by DEMATEL and used fuzzy set theory in order to incorporate imprecise judgment possibility according to linguistic comparisons. According to the prepared opinions of experts, Policy risk, knowledge asset risk, and culture risk were determined as the most crucial factors affecting the knowledge network.

Regarding the bare literature of the problem, we sought to develop a risk evaluation framework in a fuzzy environment. 
Therefore, the proposed framework may have some flaws which could be enhanced in further studies. From the view point of further studies, the investigation of the problem with respect to the three aforementioned levels in greater details can be a research direction. Furthermore, the configuration of the network can be regarded with respect to the risk sources as another future research interest. This issue can be taken into account for the circumstances in which it is required to determine the most appropriate members of the networks such that the configuration is exposed to the least possible amount of threat.

\section{References}

[1] W. Xiwei, M. Stolein, and W. Kan, "Designing knowledge chain networks in China-a proposal for a risk management system using linguistic decision making," Technological Forecasting and Social Change, vol. 77, no. 6, pp. 902-915, 2010.

[2] A. A. Kurawarwala and H. Matsuo, "Forecasting and inventory management of short life-cycle products," Operations Research, vol. 44, no. 1, pp. 131-150, 1996.

[3] R. G. McGrath and I. C. MacMillan, "How to rethink your business during uncertainty," MIT Sloan Management Review, vol. 50, no. 3, pp. 25-30, 2009.

[4] A. Agrawal and R. Henderson, "Putting patents in context: exploring knowledge transfer from MIT," Management Science, vol. 50, no. 10, pp. 1309-1318, 2004.

[5] S. Samaddar and S. S. Kadiyala, "An analysis of interorganizational resource sharing decisions in collaborative knowledge creation," European Journal of Operational Research, vol. 170, no. 1, pp. 192-210, 2005.

[6] Z. Chen, R. Chen, and S. Ma, "Knowledge chain and knowledge management," Scientific Research and Management, vol. 21, no. 1, pp. 15-18, 2002.

[7] C. W. Holsapple and M. Singh, "Knowledge chain model: activities for competitiveness," Expert Systems with Applications, vol. 20, no. 1, pp. 77-98, 2001.

[8] Z. Jiang, M. Long, and M. Zhang, "Dynamics model discussion about the knowledge supply chain and knowledge network in under industrial colony condition," Meta-Science Scientific Technology Management Journal, vol. 25, no. 7, pp. 55-60, 2004.

[9] G. T. M. Hult, D. J. Ketchen Jr., S. T. Cavusgil, and R. J. Calantone, "Knowledge as a strategic resource in supply chains," Journal of Operations Management, vol. 24, no. 5, pp. 458-475, 2006.

[10] C. W. Craighead, G. T. M. Hult, and D. J. Ketchen Jr., "The effects of innovation-cost strategy, knowledge, and action in the supply chain on firm performance," Journal of Operations Management, vol. 27, no. 5, pp. 405-421, 2009.

[11] C. Harland, R. Brenchley, and H. Walker, "Risk in supply networks," Journal of Purchasing and Supply Management, vol. 9, no. 2, pp. 51-62, 2003

[12] H. Peck, "Reconciling supply chain vulnerability, risk and supply chain management," International Journal of Logistics and Research Applications, vol. 9, no. 2, pp. 127-142, 2006.

[13] G. C. Hartmann and A. I. Lakatos, "Assessing technology risk-a case study: early identification of technology problems, coupled with early investment in problem solving, can make product delivery more predictable and reduce overall development costs," Research Technology Management, vol. 41, no. 2, pp. 32-38, 1998.
[14] M. Stößlein, "Mitigating reputational risks—a proposal with an knowledge-based stakeholder information leitstand," International Institute of Informatics and Cybernetics, vol. 1, pp. 30-35, 2009.

[15] R. Lindroth and A. Norman, "Supply chain risk and risk sharing instruments-an illustration from the telecommunication industry," in Proceedings of the 6th Annual Conference on Logistics Research Network, 2001.

[16] M. Christopher, "Supply chain vulnerability," Report for Department for Transport Local Government and Region, Cranfield University, 2002.

[17] S. S. Kulkarni, M. J. Magazine, and A. S. Raturi, "Risk pooling advantages of manufacturing network configuration," Production and Operations Management, vol. 13, no. 2, pp. 186-199, 2004.

[18] L. A. Zadeh, "Fuzzy sets," Information and Control, vol. 8, no. 3, pp. 338-353, 1965.

[19] A. Gabus and E. Fontela, World Problems, an Invitation to Further Thought within the Framework of DEMATEL, Battelle Geneva Research Centre, 1972.

[20] A. Gabus and E. Fontela, Perceptions of the World Problematique: Communication Procedure, Communicating with Those Bearing Collective Responsibility, Battelle Geneva Research Centre, 1973.

[21] G.-H. Tzeng, C.-H. Chiang, and C.-W. Li, "Evaluating intertwined effects in e-learning programs: a novel hybrid MCDM model based on factor analysis and DEMATEL," Expert Systems with Applications, vol. 32, no. 4, pp. 1028-1044, 2007.

[22] S. Opricovic and G.-H. Tzeng, "Defuzzification within a multicriteria decision model," International Journal of Uncertainty, Fuzziness and Knowlege-Based Systems, vol. 11, no. 5, pp. 635652, 2003. 


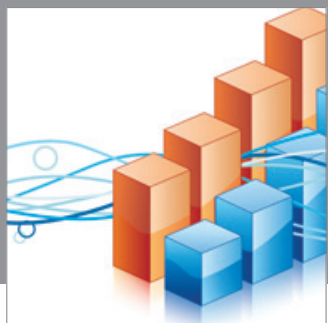

Advances in

Operations Research

mansans

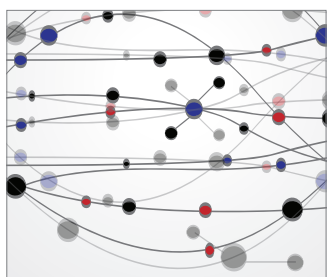

The Scientific World Journal
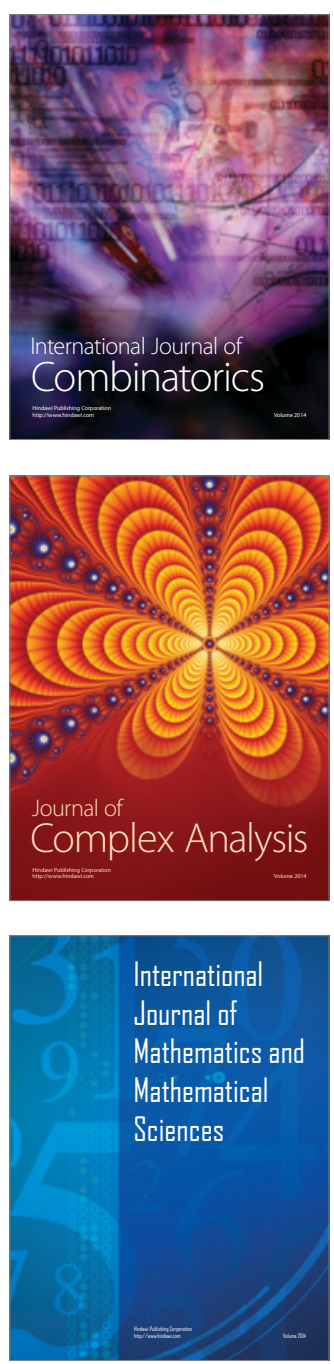
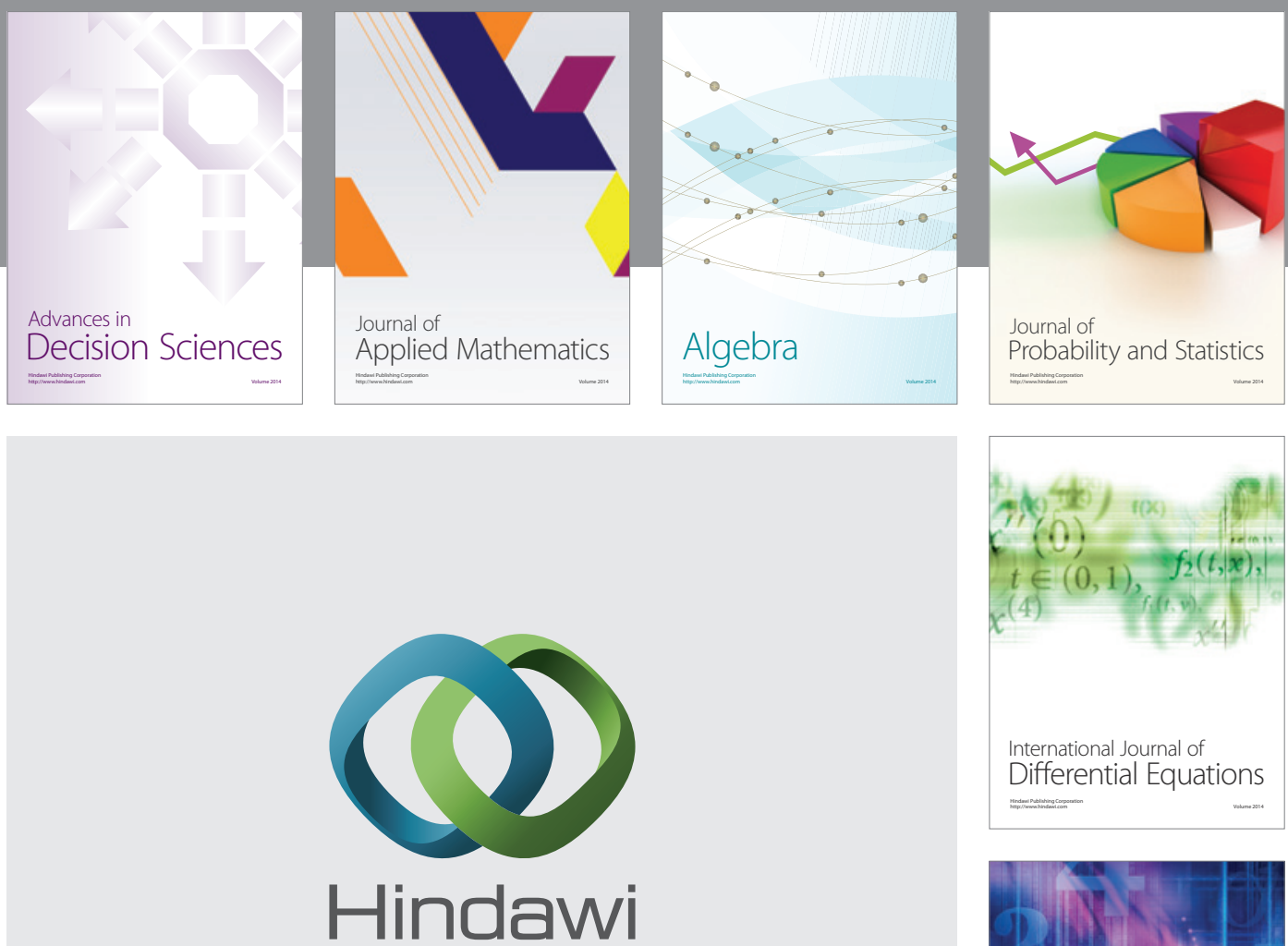

Submit your manuscripts at http://www.hindawi.com
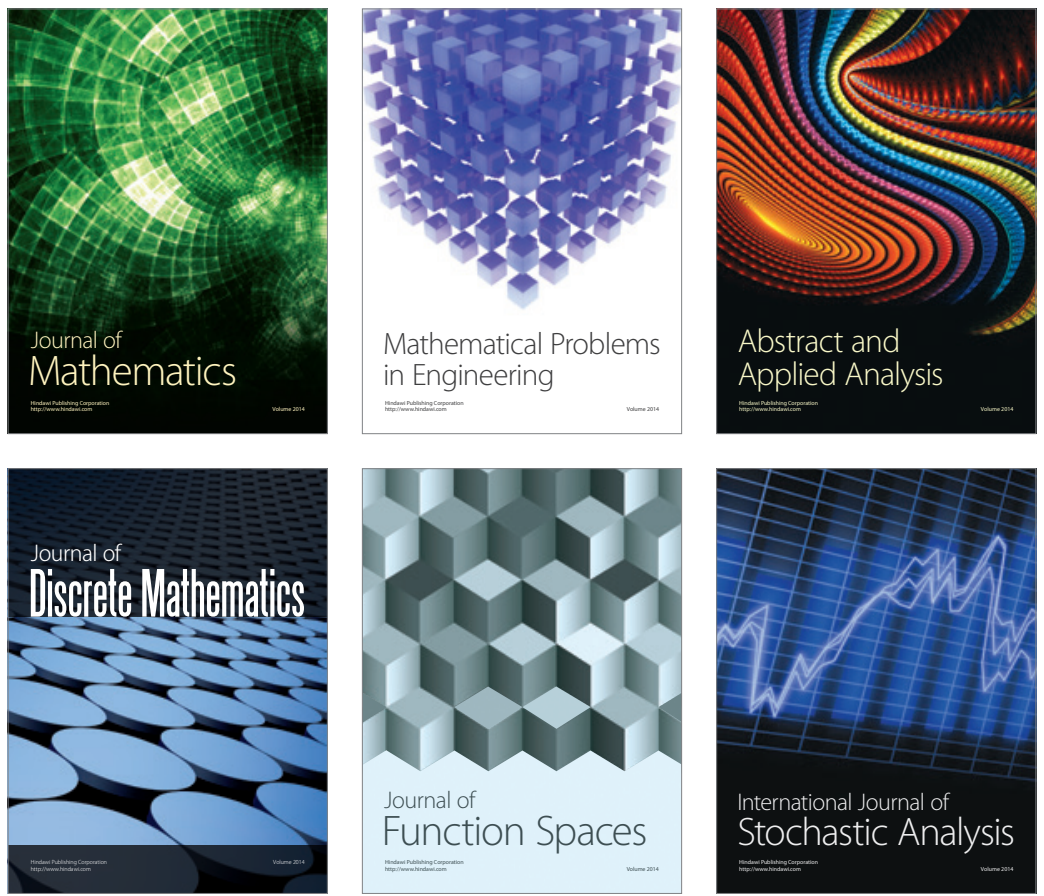

Journal of

Function Spaces

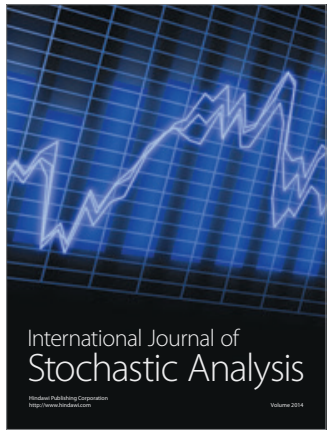

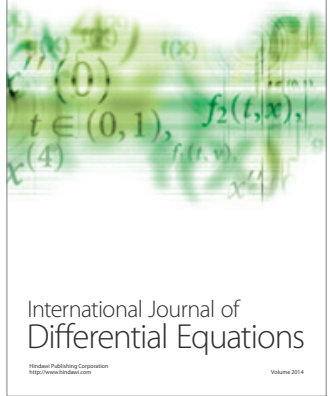
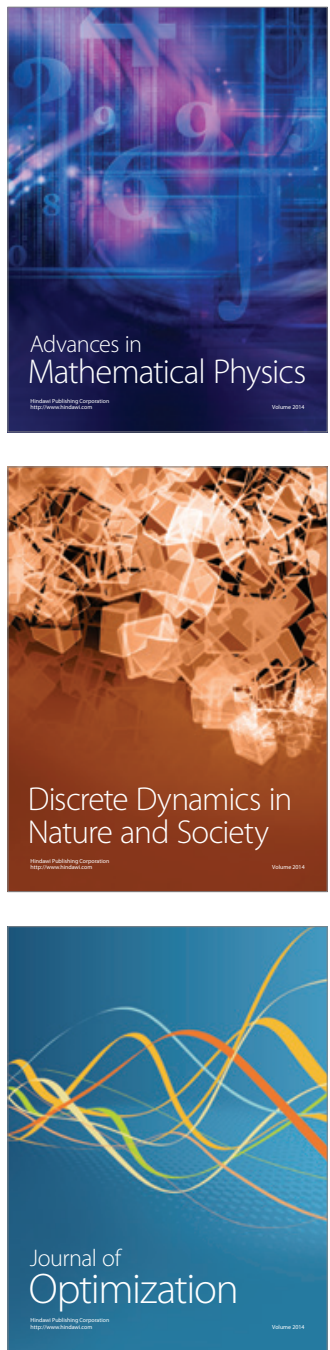